\title{
Selection and Experimental Evaluation of Universal Primers to Study the Fungal Microbiome of Higher Plants
}

\author{
Silvia Scibetta, ${ }^{1}$ Leonardo Schena, ${ }^{1, \dagger}$ Ahmed Abdelfattah, ${ }^{1,2}$ Sonia Pangallo, ${ }^{1}$ and Santa O. Cacciola ${ }^{1,3}$ \\ ${ }^{1}$ Dipartimento di Agraria, Università Mediterranea di Reggio Calabria, Località Feo di Vito, Reggio Calabria, Italy; \\ ${ }^{2}$ Department of Ecology, Environment and Plant Sciences, Stockholm University, Sweden; and ${ }^{3}$ Dipartimento di \\ Agricoltura, Alimentazione e Ambiente, Università degli Studi di Catania, Via S. Sofia 100, 95123 Catania, Italy
}

Accepted for publication 27 November 2018.

\section{ABSTRACT}

The impact of primer choice on results of metabarcoding studies was experimentally evaluated by analyzing fungal communities associated with leaves of four plant species. Significant differences in target specificity of primers were highlighted by a percentage of plant reads ranging from almost nothing to 30 to $35 \%$ of the total detected sequences. Overall, primer sets targeting the internal transcribed spacer 1 (ITS1) region proved to be more specific than those targeting the ITS2 region. A comparable taxa coverage was revealed for all investigated primer sets. However, each primer set detected only around $50 \%$ of the overall detected taxa highlighting that a consistent part of the actual fungal diversity remains undetected in studies conducted using a single couple of primers. The coverage was increased to 70 to $80 \%$ by combining results from two different primer sets. Some fungal taxa were preferentially or exclusively detected by certain primer sets and this association between primers and taxa was generally recurrent on several plant hosts. Data highlighted that a perfect set of primers to investigate the whole fungal diversity does not exist and that whatever the choice, only a fraction of the actual microbial diversity will be investigated. However, provided information may be valuable to select the best primers according to the objective of the analysis.

Additional keywords: amplicon-based metagenomics, plant pathology, primer selection.
The recent advances in high-throughput (HTS) DNA sequencing technologies have led to some fundamental breakthrough in our ability to describe, compare, and discover new microbial communities across environments (Ursell et al. 2012). In particular, the massive sequencing of polymerase chain reaction (PCR) amplicons of specific barcode DNA fragments (amplicon metagenomic or metabarcoding) is a powerful culture-independent technique. This technique is extremely useful in fungal biodiversity investigations boosting the opportunity to determine simultaneously a broad taxonomic range of organisms in terms of abundance and richness (Bálint et al. 2014; Lindahl et al. 2013). However, the metabarcoding

${ }^{\dagger}$ Corresponding author: L. Schena; E-mail: lschena@unirc.it

Funding: This research was funded by The Italian Ministry of Education, University and Research with grants "Innovazione di prodotto e di processo nelle filiera dei prodotti da forno e dolciari" (PON03PE_00090_01) and "Modelli sostenibili e nuove tecnologie per la valorizzazione delle olive e dell'olio extravergine di oliva prodotto in Calabria” (PON03PE_00090_01)-PON Ricerca e competitività 2007 to 2013

*The $\boldsymbol{e}$-Xtra logo stands for "electronic extra" and indicates that one supplementary figure and two supplementary tables are published online.

(C) 2018 The American Phytopathological Society analysis of microbial communities, from field to taxonomic composition data, involves a series of steps that may significantly influence the results. In particular, the choice of primers for the amplification of barcode regions greatly affects results and still represents an ongoing challenge in molecular ecological studies (Halwachs et al. 2017; Lindahl et al. 2013).

Currently, the internal transcribed spacers internal transcribed spacer 1 (ITS1) and ITS2 of the nuclear ribosomal DNA (rDNA) represent the primary regions to detect fungi although the need for new targets and universal primer pairs as secondary DNA barcodes has been highlighted (Schoch et al. 2012; Stielow et al. 2015; Xu 2016). Important features of the ITS region include: (i) a high number of copies per cell that facilitates the amplification of target DNA from complex environmental samples; (ii) the universality of the coding flanking regions that enables the design of universal primers to support a broad taxa coverage; and (iii) an appropriate level of variability within the ITS1 and ITS2 regions which enables a high interspecific resolution with a low intraspecific variability (Bruns et al. 1991; Schoch et al. 2012). Due to these features, ITS regions represent the most common fungal sequences in international reference datasets (NCBI, EMBL, UNITE) (Abarenkov et al. 2010; Kõljalg et al. 2013; Nilsson et al. 2009; Schoch et al. 2014). 
The alternative use of ITS1 or ITS2 region in metabarcoding studies has been questioned, with pros and cons for both fragments, but it would seem that the use of either one may lead to similar results (Blaalid et al. 2013). The amplification of the whole ITS1-5.8SITS2 region has also been proposed, but since the length of this region may range from 500 to $1,000 \mathrm{bp}$, it is difficult to cover in a single run using the currently widely used HTS technologies such as Illumina platform (Op De Beeck et al. 2014; Porter and Golding 2011).

The earliest primers for the amplification of ITS regions were published almost 30 years ago (Gardes and Bruns 1993; White et al. 1990). Since then, many additional primers have been designed in the flanking ribosomal DNA regions to enable the amplification of the target regions from most fungal lineages including the so-called early diverging lineages (Zygomycota, Chytridiomycota, and Glomeromycota) and the little-studied phylum Cryptomycota (Asemaninejad et al. 2016; Ihrmark et al. 2012; Ishii et al. 2015; Martin and Rygiewicz 2005; Mueller et al. 2016; Tedersoo et al. 2014, 2015; Toju et al. 2012; Turenne et al. 1999). Most of these primers have been used in several possible combinations to investigate soil and rhizosphere environments and, to a lesser extent, plant tissues. However, their comparative evaluation for metabarcoding analyses has been almost exclusively based on in silico studies, which enables the comparison of different primer combinations against large molecular databases (Bellemain et al. 2010; Bokulich and Mills 2013; Cheng et al. 2016; Ficetola et al. 2010; Toju et al. 2012). These studies helped in drawing a global picture of the potential specificity of the most popular rDNA primers and have also highlighted the taxonomic bias related to the nature of the fungal ITS sequences which potentially preclude the targeting of some groups of fungi. However, there is a substantial lack of comparative analyses to validate selected primers in practical experimental conditions. Furthermore, the main aim of most previous investigations was the coverage of as many fungal lineages as possible with little attention paid toward the specificity of markers for fungi. This aspect represents an important shortcoming since good primers need to be generic to amplify target DNA from most taxa, including those still unknown to the scientific community but also need to be specific enough to avoid the amplification of DNA from nontarget organisms (e.g., plant DNA).

In the present study, results of previous in silico investigations focusing on the analysis of primers for fungal metabarcoding studies were analyzed in order to identify a panel of primer sets possessing the most desirable features in terms of fungal coverage and specificity. These primer sets were comparatively and experimentally evaluated by analyzing the fungal microbiota associated with leaves of four globally ubiquitous plant crops (Vitis vinifera, Triticum aestivum, Olea europaea, and Citrus sinensis) in order to determine their performances in real experimental conditions.

\section{MATERIALS AND METHODS}

Selection of primers. A preliminary list of 30 primers was created based on data from previous studies focusing on the design and/or the in silico comparison of primers targeting various rDNA regions. These primers were evaluated according to the following criteria: (i) primers designed to target the ITS1 or ITS2 region; (ii) the universality of target region considering all currently known fungal lineages; (iii) high specificity against nontarget DNA (particularly plant DNA); and (iv) amplicon length less than $500 \mathrm{bp}$. Published data obtained with the Primer Prospector module (Walters et al. 2011) were also included for evaluating fungal taxonomic coverage of the primers. Furthermore, the number of mismatches between selected primers and corresponding target regions in the rDNA sequences of plants used in the present study (Vitis vinifera, Triticum aestivum, Olea europaea, and Citrus sinensis) were evaluated. To this aim, sequences were retrieved from GenBank, introduced in MEGA7 and aligned using MUSCLE (Kumar et al. 2016), in order to manually determine the number of mismatches. For some primers, particularly for those located on the SSU gene, this latter evaluation was not possible because of the lack of plant reference sequences.

According to preliminary analyses, eight primers were selected and coupled in six different primer sets targeting the ITS1 (set S1, S2, and S3) or the ITS2 (set S4, S5, and S6) region (Fig. 1). These
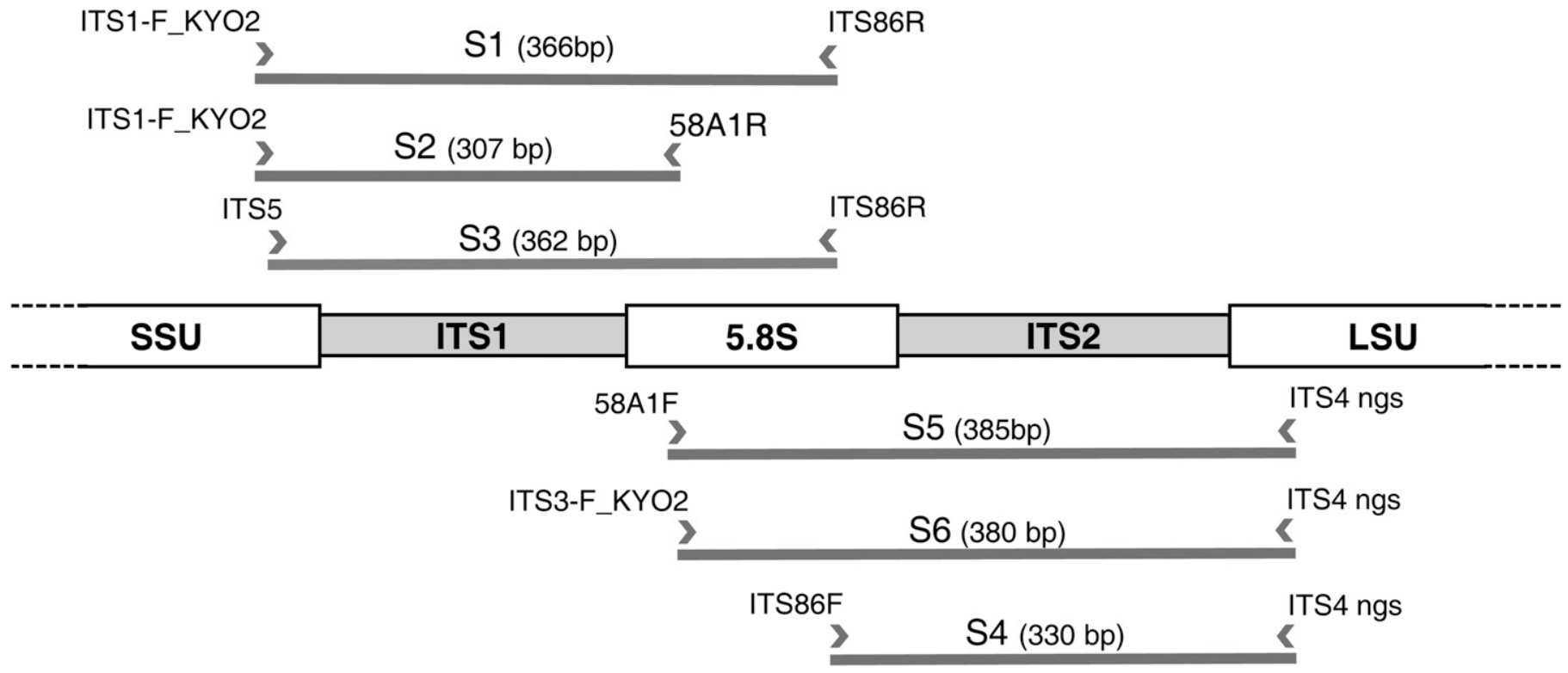

Fig. 1. Schematic representation of the internal transcribed spacer (ITS) regions of the ribosomal DNA (rDNA) and location of primers selected for metabarcoding analyses. Primers were combined in six different sets (S) to amplify either the ITS1 (S1, S2, and S3) or the ITS2 (S4, S5, and S6) region. Fragment sizes are referred to Serpula himantioides rDNA. 
primer sets were tested in experimental conditions using leaves from the four selected plant species.

DNA extraction and amplification. Fresh healthy leaves were collected in 2016 in the Gioia Tauro plane in Calabria, Southern Italy, from commercial orchards following an organic cultivation scheme. For each plant species four samples, each consisting of 20 leaves (biological replicates) were collected from 10 different plants (two leaves per plant). Collected leaves were the same age and did not show any symptom of disease or damage. Leaves were processed without any preliminary treatment of sterilization in order to keep unaltered the natural fungal population and enable the detection of both endophytes and epiphytes as well as fungal propagules in all possible forms. Sampled leaves were kept in sterile tubes at $-80^{\circ} \mathrm{C}$ and then lyophilized using a freeze dryer (Labconco Corp., Kansas City, MO). DNA extraction from freeze-dried leaves was carried out using the DNeasy Plant Mini Kit (QIAGEN, Venlo, NL) according to the manufacturer's protocol. Concentration and quality of extracted DNA were evaluated with a Nanodrop ND1000 spectrophotometer (Nano-Drop Technologies, Wilmington, $\mathrm{DE})$ and by electrophoresis through a $1.2 \%$ agarose gel in TBE buffer (90 mM Tris, $90 \mathrm{mM}$ boric acid, and $1 \mathrm{mM}$ EDTA) and Gel-red (Biotium, Hayward, CA) staining. Amplifications were performed with the selected primer sets (Fig. 1) in a total volume of $25 \mu \mathrm{l}$ containing $12.5 \mu$ of KAPA HiFi Hot Start Ready Mix (KAPA Biosystems, Wilmington, MA), $0.4 \mu \mathrm{M}$ of each primer including Illumina adaptors (https://www.illumina.com/), and $50 \mathrm{ng}$ of DNA template. Reactions were incubated in a Mastercycler gradient thermocycler (Eppendorf, Hamburg, Germany) set to have a starting denaturation of $3 \mathrm{~min}$ at $95^{\circ} \mathrm{C}$, followed by 35 cycles of $20 \mathrm{~s}$ at $98^{\circ} \mathrm{C}, 15 \mathrm{~s}$ at $56^{\circ} \mathrm{C}, 30 \mathrm{~s}$ at $72^{\circ} \mathrm{C}$, and by a final extension of $1 \mathrm{~min}$ at $72^{\circ} \mathrm{C}$. Nuclease-free water (QIAGEN) replaced template DNA in negative controls. Amplicons were visualized on gel electrophoresis at $1.2 \%$ agarose as described above.

Library preparation and sequencing. Amplicons from primer sets that enabled a consistent amplification from all plant species (S1, S3, S4, S5, and S6) were processed for the library preparation according to the NGS protocol for the MiSeq platform (Illumina Part 15044223 Rev. A) and multiplexed using the Nextera XT Kit (Illumina, San Diego, CA). The purification of PCR products and the indexing was carried out by the Agencourt AMPure XP system

TABLE 1

Summary of sequencing results obtained with the five tested primer sets (S1, S3, S4, S5, and S6) with leaves of Citrus, Olea, Triticum, and Vitis ${ }^{\mathrm{a}}$

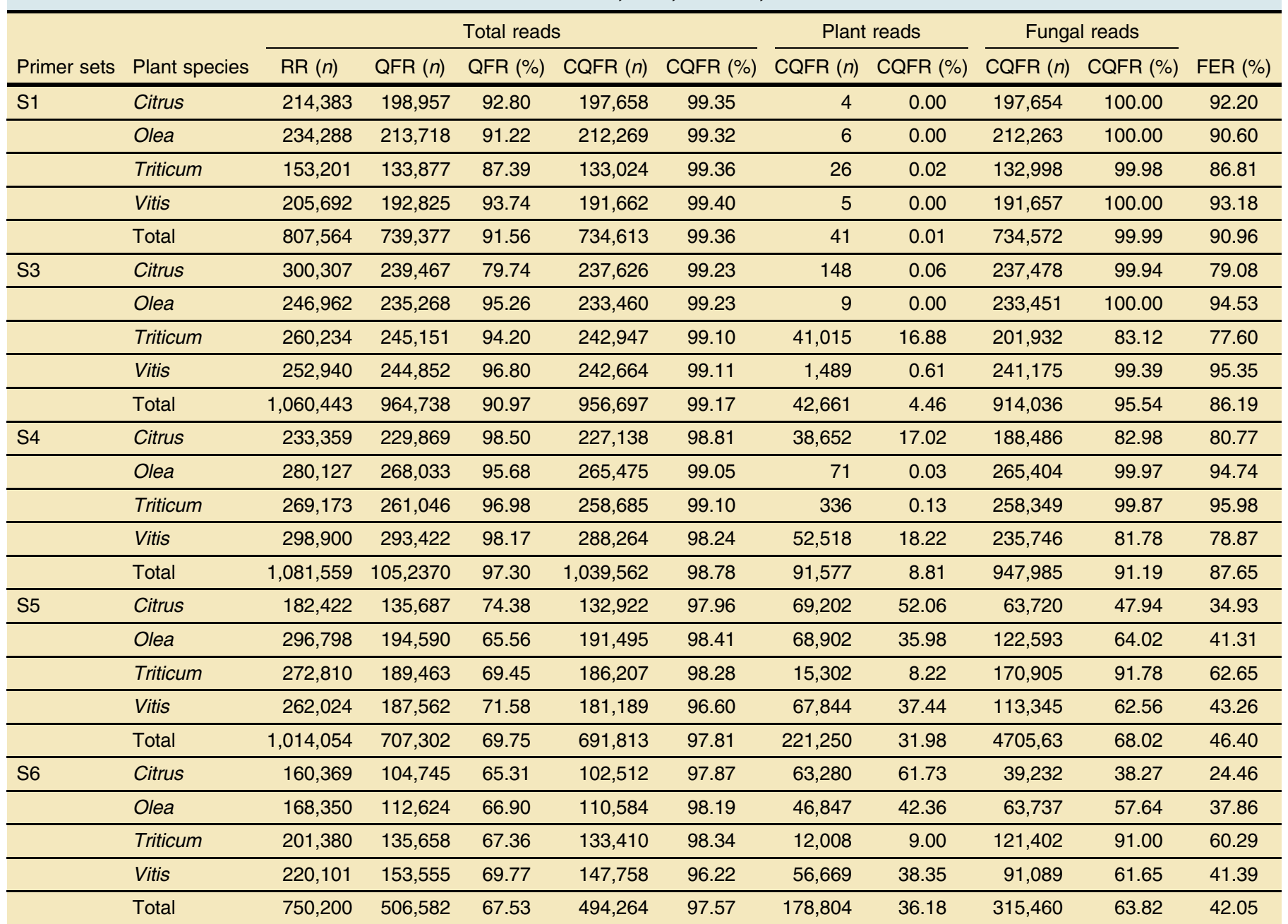

a Analyzed metrics comprised the raw reads (RR), the quality filtered reads after filtration steps (QFR), and the clustered quality filtered reads (CQFR). For each metric, absolute numbers $(n)$ and percentages $(\%)$ are reported. Furthermore, a fungal efficiency recovering (FER) index was determined to evaluate the percentage of fungal quality filtered reads compared with the total initial raw sequences. Percentages were evaluated as follows: QFR $(\%)=$ QFR $(n) / R R(n) \times 100 ;$ CQFR $(\%)=$ CQFR $(n) /$ QFR $(n) \times 100 ;$ plant CQFR $(\%)=$ plant CQFR $(n) / C Q F R(n) \times 100 ;$ fungal CQFR $(\%)=$ fungal CQFR $(n) / \operatorname{CQFR}(n) \times 100 ;$ and FER $(\%)=$ fungal CQFR $(n) / \operatorname{RR}(n) \times 100$. 
(Beckman Coulter Genomics, Brea, CA). The library was quantified by Qubit dsDNA HS fluorimetric assay (Life Technologies, Carlsbad, CA), normalized to have the same concentration in all samples, and pooled. Samples were denaturated with $0.2 \mathrm{~N} \mathrm{NaOH}$ and sequenced using Illumina $2 \times 300$ bp MiSeq Reagent Cartridge v3 in paired-end module. The PhiX control was spiked in to the final library to add base diversity (Illumina).

Bioinformatic analyses. The bioinformatics pipeline QIIME v. 1.9.1 was used to process data from HTS assay (Caporaso et al. 2010). Demultiplexed raw Illumina fastq files (NCBI-SRA accession SRP162106) were checked by FastQC to overview the quality of the reads and define the suitable filter parameters (Andrews 2010). PEAR 0.9.10 was utilized to merge paired-end reads, truncate sequences after two consecutive bases receiving a quality score $(\mathrm{Q})<20$, and delete reads containing one or more ambiguous bases or those shorter than 200 bp (Zhang et al. 2014). The de novo chimera checking was carried out using the UCHIME algorithm within VSEARCH (Rognes et al. 2016). Operational taxonomic units (OTUs) were clustered with a similarity threshold of $97 \%$ using the UCLUST-based open-reference OTU-picking workflow and the UNITE fungal ITS database released on 20 November 2016 (Abarenkov et al. 2010; He et al. 2011). Global singletons were removed and the most abundant reads in each OTU were selected as representative sequences. Unique reads were used for the taxonomy assignments using BLAST and the UNITE dynamic database (Altschul et al. 1997). Furthermore, a specific index designated here as fungal efficiency recovering (FER, \%), expressing the percentage of quality fungal reads compared with the total original raw sequences, was utilized to summarize the performance of the primers. The OTU table was rarefied at an even sequencing depth of 7,220 reads/sample. For each primer/ host combination, the alpha-diversity was evaluated using OTU richness, Shannon index, and Simpson index and the results were statistically compared using a nonparametric two-sample $t$ test. Furthermore, the Kruskal-Wallis nonparametric test was carried out to identify taxa with significantly different relative abundance (RA), by considering only OTUs with a minimum average RA of $0.05 \%$. These data were visualized by constructing a heatmap for each plant. In both analyses, the raw $P$ values were calculated through 1,000 Monte Carlo permutations and corrected using the false discovery rate (FDR) procedure. The corrected FDR $P$ values of $<0.05$ were considered significant. Finally, a matrix of shared OTUs was generated to determine the percentage of fungal OTUs detected with individual or couple of primer sets compared with OTUs cumulatively detected with the five primer pairs.

\section{RESULTS}

Selection of primers and amplifications. In silico data recognition of published rDNA primers within the preliminary list

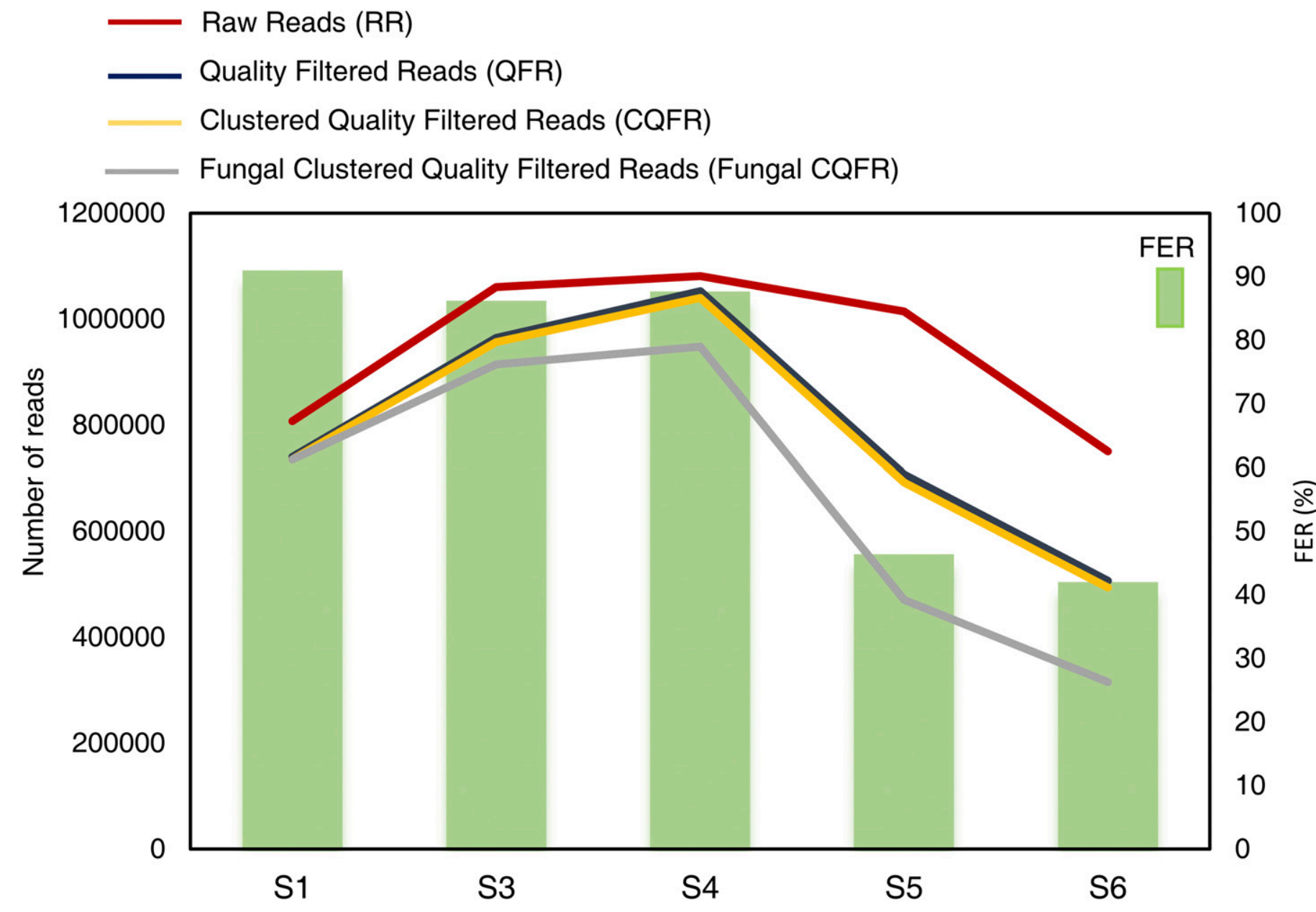

Fig. 2. Overall performance of the five selected primer sets with leaves of the four investigated plant species (Vitis vinifera, Triticum aestivum, Olea europaea and Citrus sinensis) in terms of detected number of reads (lines, left axis) and FER, fungal efficiency recovering percentage (columns, right axis). 
showed a lower risk of coamplification of plant DNA for primers targeting the SSU gene as compared with $5.8 \mathrm{~S}$ and LSU primers. The 5.8S primers had a fungal coverage comparable to those of SSU primers and a higher plant coamplification percentage. The LSU primers were characterized by a slightly lower fungal coverage and a higher percentage of plant coamplification. Primers ITS5 and ITS1-F_KYO2, targeting the SSU gene, were selected for their potential in reducing the coamplification of plant DNA $(<10 \%)$ and the high coverage of Ascomycetes ( $>95 \%)$, Basidiomycetes $(>92 \%)$, and early diverging fungi ( $>94 \%$ ) (Supplementary Table S1; Fig. 1). Among the 5.8S primers, 58A1F was selected for its low coamplification of plant DNA $(<28 \%)$ and for the presence of two (grape) and one (wheat and orange) mismatches with corresponding target DNA in plants. This primer was also firstly utilized in the present study as reverse primer (58A1R). On the other hand, primer ITS3_KYO2 was selected for having the highest fungal coverage for Ascomycetes (>99\%), Basidiomycetes (>98\%), and early diverging fungi ( $>99 \%)$. Furthermore, despite the absence of wide in silico data on the coverage of primers ITS86F and ITS86R, they were selected because of the reported good performance in metabarcoding analyses focusing on soilborne fungi (Op De Beeck et al. 2014; Waud et al. 2014). In addition, these primers showed, globally, high number of mismatches with the plant species evaluated in the present study. Among primers targeting the LSU gene, the ITS4ngs was preferred since it includes the original ITS4 primer and three variants of this primer, designed to have a higher fungal coverage including Ascomycetes (>96\%), Basidiomycetes (>97\%), and early diverging fungi (>93\%) (Tedersoo et al. 2014).

The eight selected primers were combined to have six primer sets targeting the ITS1 (set S1, S2, and S3) or the ITS2 (set S4, S5, and S6) region and enabled the amplification of DNA fragments of the expected size from leaves of $V$. vinifera, T. aestivum, O. europaea, and $C$. sinensis. These fragments ranged from 300 to 385 bp considering Serpula himantioides as reference fungal species (Fig. 1). Among the six tested primer sets, only set S2 did not enable a consistent amplification from all plant species and was excluded from the HTS validation experiments.

Specificity of tested primers. A total of 5,774,522 raw reads were obtained by Illumina MiSeq sequencing from the pooled amplicon library of 80 samples (five primer sets, four plant species, and four biological replicates). The total number of raw reads per plant species ranged from 750,200 (set S6) to more than one million (sets S3, S4, and S5) considering cumulatively the four plant species (Table 1; Fig. 2). Significant differences were also observed in terms of quality filtered reads (QFR). The best performance in terms of percentage of QFR was determined for set S4 (97.3\%) targeting the ITS2 region, followed by sets S1 (91.6\%) and S3 (91.0\%) targeting the ITS1 region (Table 1). Much lower percentages of QFR were revealed for sets S5 (69.7\%) and S6 (67.5\%), targeting the ITS2 region. None of the analyzed reads was flagged as chimera. After quality and chimera filtering, 3,970,369 reads were clustered at $97 \%$ similarity threshold. Furthermore, after the removal of singletons, 7,726 OTUs, corresponding to 3,916,949 reads were retained. Considering all primer sets, 3,382,616 clustered quality filtered reads $(86.3 \%)$ were assigned to fungal lineages after the removal of plant sequences (Table 1).

Primers S1 and S3, targeting the ITS1 regions, showed a significantly higher FER (91.0 and $86.2 \%$, respectively) compared with sets S5 and S6, targeting the ITS2 (46.4 and $42.1 \%$, respectively). In contrast, set S4 showed a level of FER $(87.7 \%)$ comparable to that of ITS1 primers. In agreement with these results, the coamplification of plant DNA was significantly lower for primer

\section{Fungal OTUs — Plant OTUs}

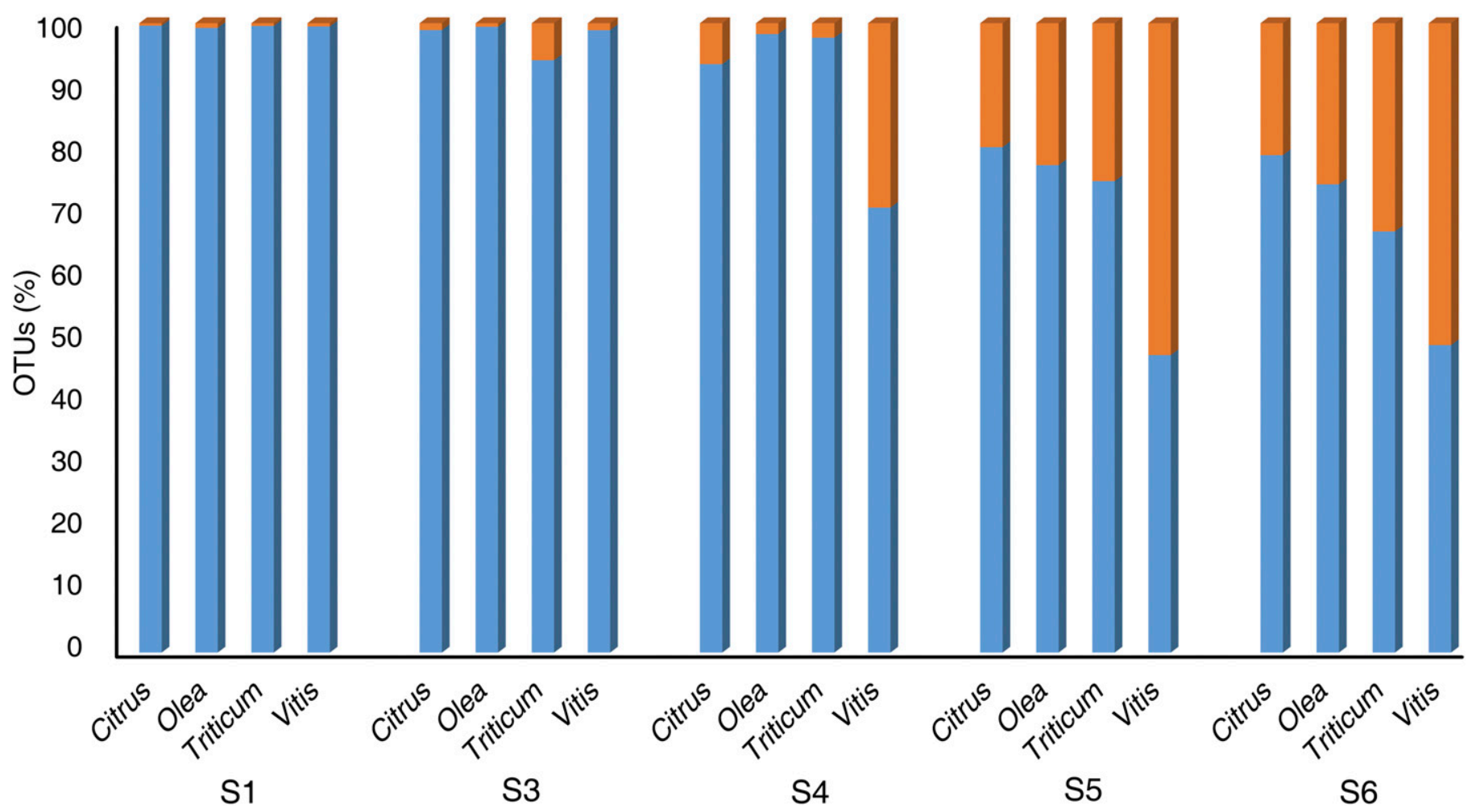

Fig. 3. Percentage of total fungal (blue bars) and plant (orange bars) OTUs recovered from Citrus, Olea, Triticum, and Vitis with primer sets S1, S3, S4, S5, and S6. 
sets S1, S3, and S4 (0.01, 4.46, and $8.81 \%$, respectively) and much higher for sets S5 (31.98\%) and S6 (36.18\%) (Table 1; Fig. 2). In addition, almost all OTUs detected with primer sets S1, S3, and S4 from Citrus, Olea, and Triticum were assigned to fungal taxa (Fig. $3)$. An exception was represented by primer set $\mathrm{S} 4$ that detected a quite high percentage of plant OTUs (29.3\%) from Vitis. A much higher percentage of plant OTUs was detected with primer sets S5 and S6 from all hosts and in particular from Vitis (Fig. 3).

Fungal taxa coverage. For all investigated primer/host combinations rarefaction curves generated profiles tending toward saturation indicating that at the selected sequencing depth the great majority of OTUs had been captured (Supplementary Fig. S1). Overall, a total of 528 OTUs were assigned to fungal lineages identified at different levels, from phylum to genus. Among these, $332,260,186$, and 250 fungal OTUs were detected in the phyllosphere of Vitis, Citrus, Olea, and Triticum species, respectively (Table 2; Supplementary Table S2. However, the two-sample $t$ test analysis based on the number of observed OTUs, Shannon index, and Simpson index did not reveal any significant difference between the primer sets in all plant species (Table 2).

Results showed that only a fraction of the actual genetic diversity can be detected with a single primer set. In particular, the number of fungal OTUs detected from each host with individual primer pairs was around $50 \%$ of those cumulatively detected with the five primer sets (Table 3). The best individual sets were S5 for Citrus $(58.7 \%)$ and Vitis (50.8\%), S6 for Olea (62.3\%), and S3 for Triticum

TABLE 2

Alpha-diversity indexes (observed operational taxonomic units [OTUs], Shannon, and Simpson) evaluated per each plant species and primer set at an even sequencing depth of 7,220 reads/sample

\begin{tabular}{|c|c|c|c|c|}
\hline $\begin{array}{l}\text { Plant } \\
\text { species }\end{array}$ & $\begin{array}{l}\text { Primer } \\
\text { sets }\end{array}$ & $\begin{array}{c}\text { Number of } \\
\text { observed OTUs }\end{array}$ & $\begin{array}{c}\text { Shannon } \\
\text { index }\end{array}$ & $\begin{array}{c}\text { Simpson } \\
\text { index }\end{array}$ \\
\hline \multirow[t]{5}{*}{ Citrus } & S1 & 182 & 4,200 & 0.872 \\
\hline & S3 & 177 & 4,269 & 0.883 \\
\hline & S4 & 185 & 4,960 & 0.923 \\
\hline & S5 & 195 & 4,698 & 0.897 \\
\hline & S6 & 179 & 4,410 & 0.876 \\
\hline Total & & 332 & & \\
\hline \multirow[t]{5}{*}{ Olea } & S1 & 125 & 3,735 & 0.843 \\
\hline & S3 & 124 & 3,796 & 0.852 \\
\hline & S4 & 141 & 4,244 & 0.887 \\
\hline & S5 & 148 & 4,242 & 0.888 \\
\hline & S6 & 162 & 3,875 & 0.844 \\
\hline Total & & 260 & & \\
\hline \multirow[t]{5}{*}{ Triticum } & S1 & 87 & 4,010 & 0.893 \\
\hline & S3 & 93 & 4,113 & 0.899 \\
\hline & S4 & 77 & 4,166 & 0.906 \\
\hline & S5 & 89 & 3,126 & 0.719 \\
\hline & S6 & 86 & 2,414 & 0.602 \\
\hline Total & & 186 & & \\
\hline \multirow[t]{5}{*}{ Vitis } & S1 & 120 & 3,427 & 0.848 \\
\hline & S3 & 108 & 3,454 & 0.850 \\
\hline & S4 & 125 & 3,893 & 0.883 \\
\hline & S5 & 127 & 3,648 & 0.856 \\
\hline & S6 & 118 & 3,100 & 0.733 \\
\hline Total & & 250 & & \\
\hline
\end{tabular}

$(50.0 \%)$ (Table 3). The coverage of detected OTUs was improved by cumulatively considering OTUs obtained with two primers sets (Table 3). Overall, the best results were obtained combining primers sets targeting the ITS1 region with those targeting ITS2 region. For instance, the percentage of detected OTUs increased to $78.6 \%$ with primers S1 and S4 for Citrus, $72.8 \%$ with primers S1 and S6 for Vitis, and 79.2 and $72.0 \%$ with primers S3 and S6 for Olive and Triticum, respectively (Table 3).

Fungal community structure. Considering all investigated hosts and primer sets, Ascomycota was the most abundant phylum representing around $79 \%$ of the detected sequences, followed by Basidiomycota (20\%), unidentified Fungi $(0.68 \%)$, Zygomycota $(0.1 \%)$, Glomeromycota $(0.01 \%)$, fungi Incertae sedis $(0.002 \%)$, and Chytridiomycota $(0.001 \%)$ (Fig. 4). The five investigated primer sets detected a higher RA of Ascomycota on Olea $(90 \%)$ and Vitis (84.3\%) compared with Citrus (66.5\%) and Triticum $(75.5 \%)$ (Fig. 4). In these two latter hosts, the lower abundance of Ascomycota was largely compensated by Basidiomycota. Overall, the RA of Ascomycota and Basidiomycota detected with different sets was similar on Citrus, Olea, and Vitis. On the contrary, on Triticum, primer sets S1, S3, and S4 detected a much higher concentration of Basidiomycota compared with primer sets S5 and S6 (Fig. 4). In particular, the RA of Basidiomycota was 4 and $6.4 \%$ with primer sets S5 and S6, and increased to 34.9, 36.6, and 38.2\% with primer sets S1, S3, and S4, respectively. The phylum Zygomycota was mainly detected on Olea with a variable RA according to the primer sets (Fig. 4).

At the genus level, most of the taxa were detected with a very low RA (Fig. 5). In the case of Vitis and Triticum, 11 and 7 taxa had an $\mathrm{RA} \geq 1 \%$ and represented around $90 \%$ of the total fungal reads. Similarly, in Citrus and Olea, 15 and 13 taxa had an RA $\geq 1 \%$ and

TABLE 3

Percentage of operational taxonomic units (OTUs) detected from each host with individual primer sets $\left({ }^{*}\right)$ or combining two different primer sets compared with the total number of OTUs cumulatively detected with the five primer pairs

\begin{tabular}{|c|c|c|c|c|c|c|}
\hline Primer set & S1 & S3 & S4 & S5 & S6 & Species \\
\hline \multirow[t]{4}{*}{ S1 } & $54.8^{*}$ & 66.2 & 78.6 & 77.0 & 76.5 & Citrus \\
\hline & $48.1^{*}$ & 60.0 & 72.7 & 74.6 & 78.5 & Olea \\
\hline & $46.7^{\star}$ & 63.4 & 63.0 & 68.9 & 67.7 & Triticum \\
\hline & $48.0^{*}$ & 58.8 & 70.8 & 72.0 & 72.8 & Vitis \\
\hline \multirow[t]{4}{*}{ S3 } & & $53.3^{*}$ & 76.5 & 75.9 & 73.5 & Citrus \\
\hline & & $47.7^{*}$ & 71.1 & 71.9 & 79.2 & Olea \\
\hline & & $50.0^{*}$ & 66.1 & 69.9 & 72.0 & Triticum \\
\hline & & $43.2^{*}$ & 65.6 & 65.2 & 65.2 & Vitis \\
\hline \multirow[t]{4}{*}{ S4 } & & & $55.7^{*}$ & 72.0 & 69.0 & Citrus \\
\hline & & & $54.2^{*}$ & 71.5 & 73.0 & Olea \\
\hline & & & $41.4^{*}$ & 61.8 & 60.2 & Triticum \\
\hline & & & $50.0^{*}$ & 72.4 & 68.0 & Vitis \\
\hline \multirow[t]{4}{*}{ S5 } & & & & $58.7^{*}$ & 69.3 & Citrus \\
\hline & & & & $56.9^{*}$ & 77.3 & Olea \\
\hline & & & & $47.8^{*}$ & 64.5 & Triticum \\
\hline & & & & $50.8^{*}$ & 68.4 & Vitis \\
\hline \multirow[t]{4}{*}{ S6 } & & & & & $53.9^{*}$ & Citrus \\
\hline & & & & & $62.3^{*}$ & Olea \\
\hline & & & & & $46.2^{*}$ & Triticum \\
\hline & & & & & $47.2^{*}$ & Vitis \\
\hline
\end{tabular}


represented around $80 \%$ of the reads (Fig. 5). The number of taxa detected with a significant difference using the five primer sets was 21, 12, 11, and 12 for Citrus, Olea, Triticum, and Vitis, respectively (Fig. 6). Within them, taxa like Cladosporium, Didymella, Ustilaginales, and Epicoccum were favored by primer sets targeting the ITS1 region (S1 and S3), regardless of the host. Conversely, other taxa like Mycosphaerella, Togniniella, Celerioriella, and Pseudocercospora were preferentially detected with primer sets targeting the ITS2 region (S4, S5, and S6) (Fig. 6). Particularly relevant was the case of the genus Puccinia, which was detected on Triticum almost exclusively with primer sets S1, S3, and S4. Conversely, these primer sets did not detect (as in the case of S1), or detected with a very small RA (as in the case of S3 and S4), the genus Togniniella, which was the most abundant taxon with sets S5 and S6 (Fig. 6). The genus Capnodium had quite a high RA in Citrus but was exclusively detected with primer sets S4, S5, and S6 (Fig. 6).

\section{DISCUSSION}

A number of studies, mainly conducted using in silico approaches, have highlighted that the selection of primers for metabarcoding analyses is the variable that most affects final outputs and represents a major challenge in future analyses of microbial communities. In the present study, experimental trials were specifically conducted to practically evaluate these issues focusing the attention on epiphytic fungal communities associated with leaves of four different plant species. The obtained results highlighted a number of challenges specifically related to the investigated system that can be easily extended to many other environmental systems including plant, water, soil, air and animals associated microbial populations. Furthermore, issues highlighted in the present study for the investigation of fungal populations are likely to exist in a similar manner for other important microbial kingdoms, including bacteria. We paid great attention in the preliminary selection of primers to be used in our system by analyzing currently available data with the overall aims of increasing specificity for fungi, i.e., to maximize the fungal coverage and reduce the cross reaction with unwanted plant DNA. The five selected primer sets are among the best currently available for fungal metabarcoding analyses based on the use of ITS1 and/or ITS2 regions. Although, it cannot be excluded that some valuable primers were discarded in the present study and that better primers could be identified by future studies, the overall results clearly highlighted that a perfect primer set to investigate the whole fungal diversity does not exist.

All primer sets selected in the present study proved to be appropriate for metagenomic analyses. In fact, for all primer/host combinations, rarefaction curves tended to saturation indicating that fungal diversity was properly captured by all primer sets. A good performance was also confirmed by the very few singletons and by the absence of chimeras which may represent serious obstacles in metabarcoding analyses (Ihrmark et al. 2012; Taylor et al. 2014, 2016). Nevertheless, significant differences in terms of specificity were highlighted by a percentage of plant reads ranging from almost nothing to 30 to $35 \%$ of the total detected sequences. Primer sets targeting the ITS1 region proved to be more specific compared with those targeting the ITS2 region and this result was consistent with all plant species, although more evident for $V$. vinifera. The higher specificity of primer sets targeting the ITS1 region was the consequence of the higher specificity of primers designed on the SSU gene, although also primer ITS $86 \mathrm{~F}$, targeting the $5.8 \mathrm{~S}$ gene, proved specific for fungi. In fact, this last primer showed high specificity as reverse primer to amplify the ITS1 region (sets S1 and S3) and as forward primer to amplify the ITS2 region (set S4). Conversely, other primers targeting the $5.8 \mathrm{~S}$ gene (58A1F and ITS3-F_KYO2) proved much less specific considering the high percentage of plant

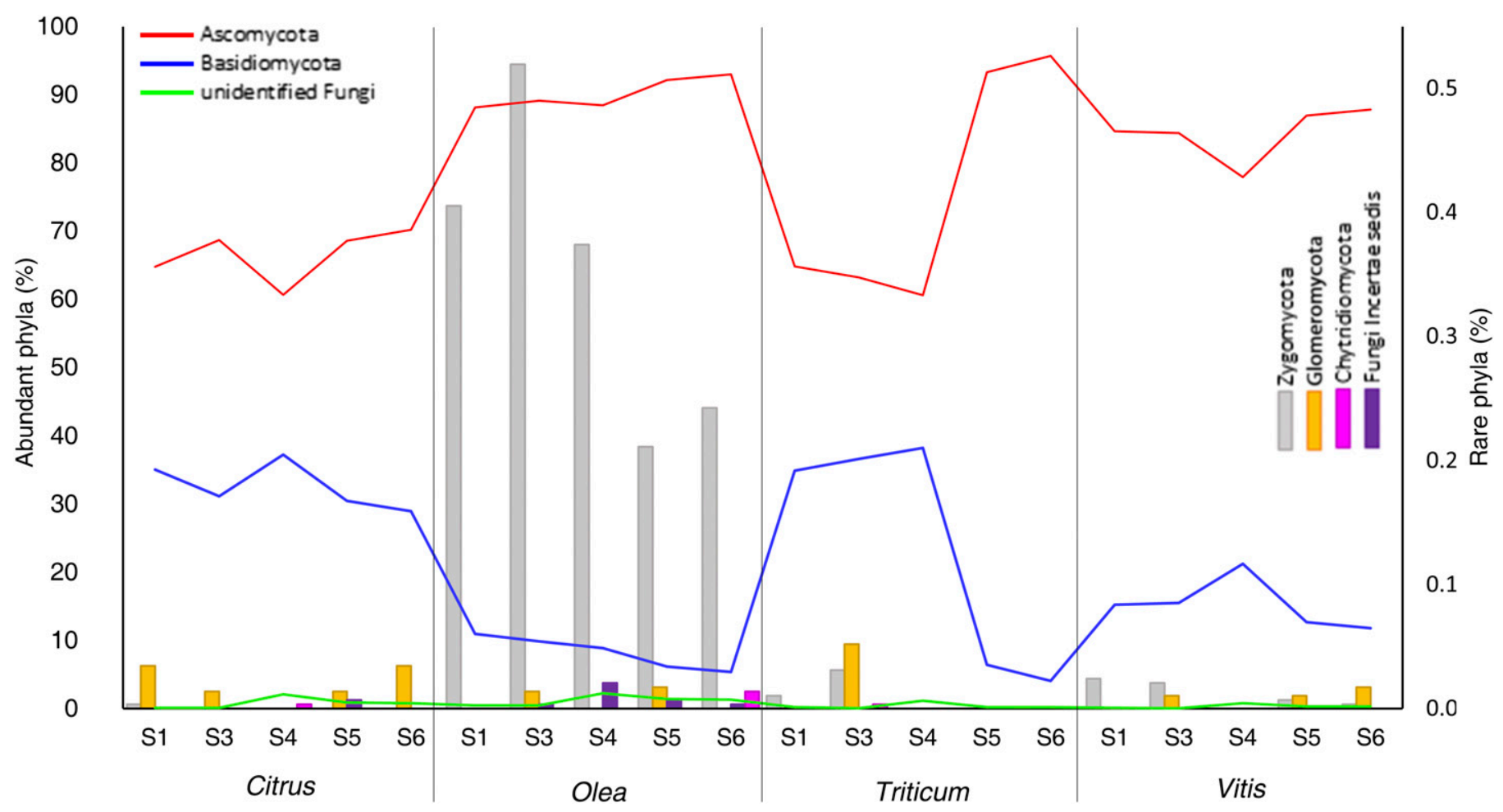

Fig. 4. Relative abundance (RA) of fungal phyla detected from Citrus, Olea, Triticum, and Vitis with primer sets S1, S3, S4, S5, and S6. Horizontal lines refers to abundant phyla (Ascomycota, Basidiomycota, and unidentified fungi) and their RA is plotted on the left axis. Rare phyla (Zygomycota, Chytridiomycota, Glomeromycota, and fungi Incertae sedis) are represented by columns and their RA is plotted on the right axis. 
reads obtained in combination with primer ITS4-ngs, which is known to amplify target DNA from plants (Cheng et al. 2016; Toju et al. 2012).

Despite the above-mentioned differences in terms of specificity, this feature did not affect results in terms of the number of recovered fungal taxa. Indeed, a comparable taxa coverage was revealed for all selected primer sets on all the plant hosts regardless of their specificity. However, although primer sets behaved in a similar manner in terms of number of detected taxa, each set did not enable the detection of a consistent part of the actual fungal diversity. Indeed, each primer set detected around $50 \%$ of the taxa cumulatively detected with the five primer sets. We also demonstrated that the coverage was increased to 70 to $80 \%$ by combining results from two different primer sets, especially when ITS1 primers were coupled with primers targeting the ITS2 region. These results clearly indicate that most of the currently available metabarcoding studies have only revealed a fraction of the fungal diversity since a single set of primers has been used in most investigations. Furthermore, our results reinforce the perception that even when using more primer sets a large part of the diversity is likely to remain undiscovered, although metagenomic analyses have greatly increased our ability to investigate complex populations (Op De Beeck et al. 2014; Orgiazzi et al. 2012). According to a 2016 estimation from the Dictionary of Fungi (http://www.speciesfungorum.org/),
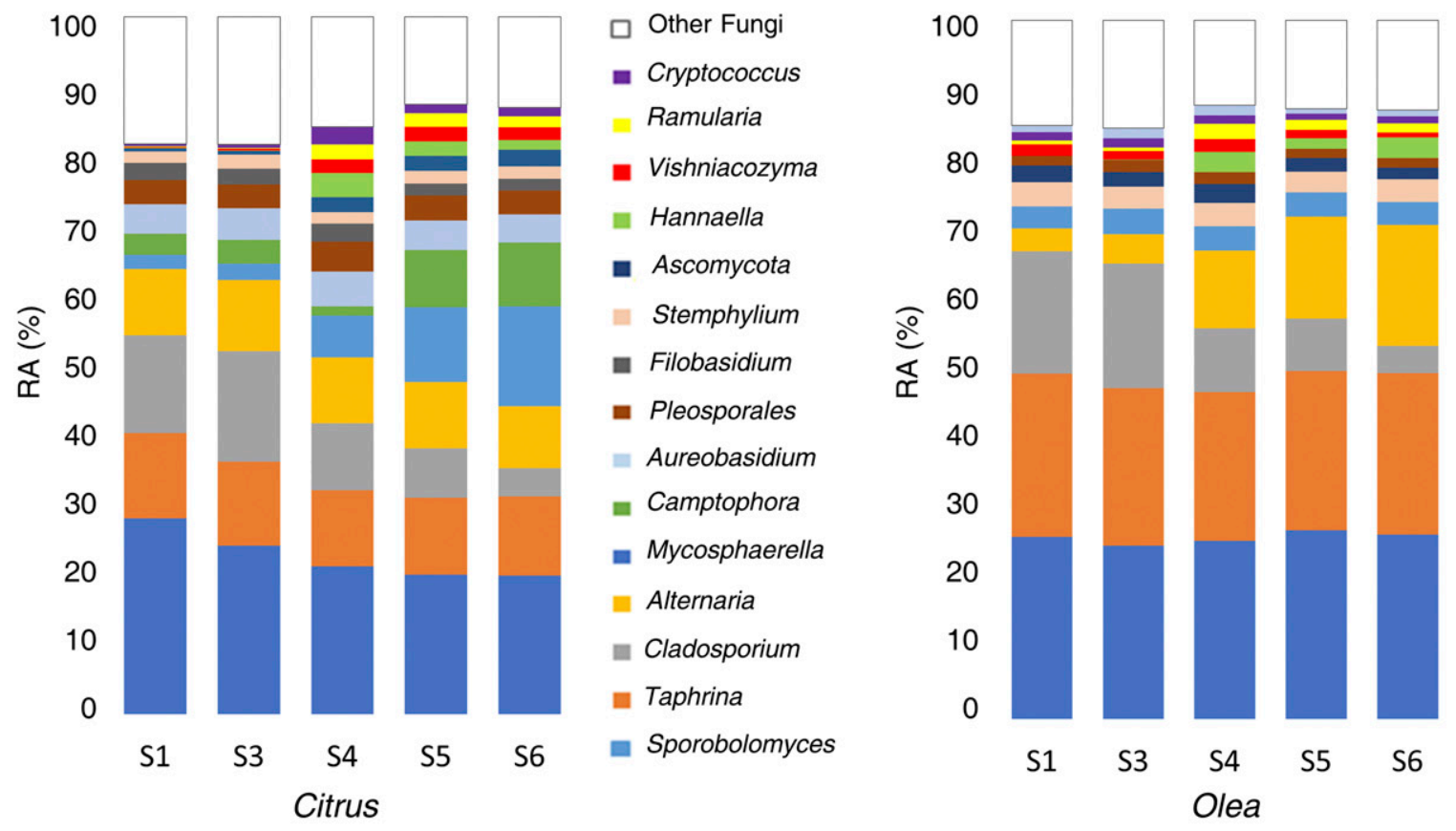

$\square$ Other Fungi

Filobasidium

- Venturiales

unidentified Fungi

- Erythrobasidium

- Pseudocercospora

- Neurospora

- Sporobolomyces

- Pleospora

- Stemphylium

mycosphaerella

- Cladosporium

n Alternaria

- Aureobasidium
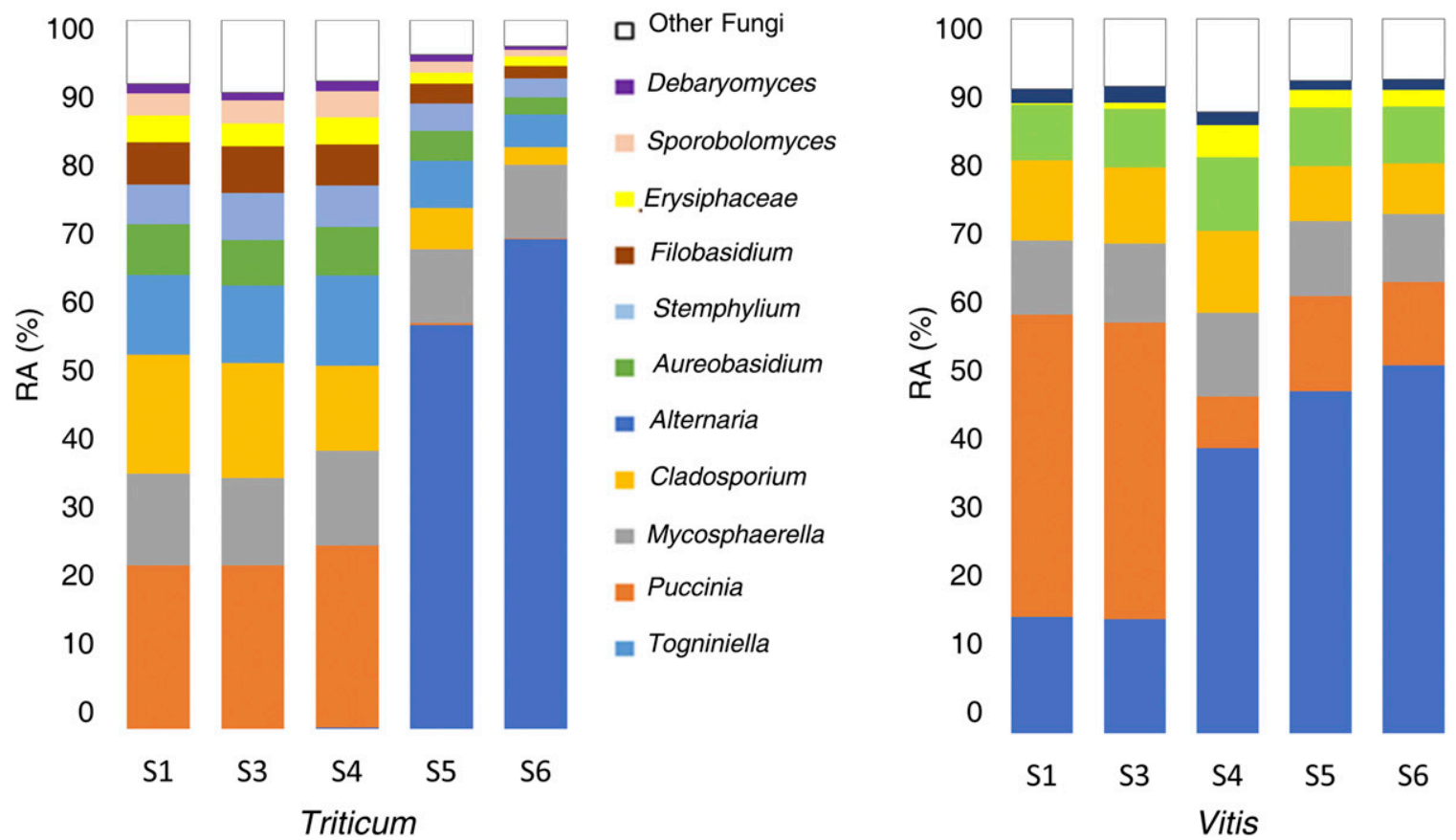

$\square$ Other Fungi

- Botrytis

Vishniacozyma

= Alternaria

- Sporobolomyces

an Stemphylium

n Cladosporium

- Mycosphaerella

Fig. 5. Fungal taxa with a relative abundance $(\mathrm{RA}) \geq 1 \%$, detected with each investigated primer set (S1, S3, S4, S5, and S6) in leaves of Citrus, Olea, Triticum, and Vitis. Fungal taxa with an RA $\leq 1 \%$ were cumulatively reported as "other fungi." 
approximately 130,000 fungal taxa have been so far described versus an estimated diversity of 1.5 to 6 million (Blackwell 2011; Hawksworth 1991; Taylor et al. 2014). Excluding arbuscular mycorrhizas, most fungal barcode primers target the ITS regions of the rDNA. Consequently, the existence of fungal lineages completely missed by these markers cannot be excluded also because most of the currently available sequences are from cultivable fungi and this, indeed, represents a limit for the design of universal primers.

In agreement with the above results the analysis of the taxonomic composition of fungal populations associated with the leaves of the four investigated plant species revealed heterogeneous and complex results significantly influenced by the utilized primer sets. At phylum

\section{Citrus}

\begin{tabular}{|c|c|c|c|c|c|}
\hline \multirow{2}{*}{ Taxon } & \multicolumn{5}{|c|}{ Relative abundance } \\
\hline & S1 & S3 & S4 & S5 & S6 \\
\hline Unidentified Fungi & $0,128 \%$ & $0,139 \%$ & $2,109 \%$ & $0,928 \%$ & $0,796 \%$ \\
\hline Lignincola & $1,807 \%$ & $1,170 \%$ & & & \\
\hline Dothideomycetes & $0,197 \%$ & $0,173 \%$ & $0,277 \%$ & $1,288 \%$ & $1,250 \%$ \\
\hline Mycosphaerellaceae & $2,022 \%$ & $2,175 \%$ & $0,035 \%$ & $0,035 \%$ & $0,014 \%$ \\
\hline Malassezia & $0,229 \%$ & $0,104 \%$ & & $0,007 \%$ & $0,007 \%$ \\
\hline Dictyosporium & $1,745 \%$ & $1,731 \%$ & & & \\
\hline Cryptococcus & $0,301 \%$ & $0,509 \%$ & $2,524 \%$ & $1,257 \%$ & $1,288 \%$ \\
\hline Cladosporium & $13,996 \%$ & $15,821 \%$ & $9,581 \%$ & $7,067 \%$ & $4,024 \%$ \\
\hline Didymella & $0,637 \%$ & $0,772 \%$ & $0,007 \%$ & $0,003 \%$ & \\
\hline Mycosphaerella & $2,012 \%$ & $2,351 \%$ & $6,015 \%$ & $10,717 \%$ & $14,269 \%$ \\
\hline Epicoccum & $1,437 \%$ & $1,707 \%$ & $0,003 \%$ & $0,017 \%$ & $0,093 \%$ \\
\hline Pseudocercospora & $0,021 \%$ & $0,042 \%$ & $0,589 \%$ & $0,204 \%$ & $0,665 \%$ \\
\hline Exobasidiomycetes & $0,035 \%$ & $0,170 \%$ & $0,876 \%$ & $0,436 \%$ & $0,533 \%$ \\
\hline Ascomycota & $0,402 \%$ & $0,474 \%$ & $2,105 \%$ & $2,147 \%$ & $2,448 \%$ \\
\hline Hannaella & $0,076 \%$ & $0,080 \%$ & $3,501 \%$ & $2,088 \%$ & $1,392 \%$ \\
\hline Ramularia & $0,121 \%$ & $0,118 \%$ & $2,157 \%$ & $1,936 \%$ & $1,569 \%$ \\
\hline Ustilaginales & $0,312 \%$ & $0,253 \%$ & & & \\
\hline Capnodiales & $1,620 \%$ & $1,918 \%$ & $0,055 \%$ & $0,083 \%$ & $0,048 \%$ \\
\hline Bulleromyces & $0,274 \%$ & $0,280 \%$ & $0,461 \%$ & $0,190 \%$ & $0,277 \%$ \\
\hline Celerioriella & & & $0,087 \%$ & $0,045 \%$ & $0,104 \%$ \\
\hline Capnodium & & & $1,908 \%$ & $1,354 \%$ & $1,489 \%$ \\
\hline
\end{tabular}

\section{Triticum}

\begin{tabular}{l|rrrrc}
\hline \multirow{2}{*}{ Taxon } & \multicolumn{5}{c}{ Relative abundance } \\
\cline { 2 - 6 } Cystofilobasidium & S3 & S4 & S5 & S6 \\
\hline Didymella & $0,557 \%$ & $0,454 \%$ & $0,048 \%$ & $0,045 \%$ & $0,038 \%$ \\
Togniniella & $0,970 \%$ & $0,942 \%$ & & $0,003 \%$ & \\
Cryptococcus & & $0,003 \%$ & $0,229 \%$ & $56,991 \%$ & $69,120 \%$ \\
Unidentified Fungi & $0,128 \%$ & $0,301 \%$ & $1,361 \%$ & $0,744 \%$ & $0,478 \%$ \\
Cladosporium & $0,235 \%$ & $0,097 \%$ & $1,160 \%$ & $0,235 \%$ & $0,218 \%$ \\
Dothideomycetes & $16,790 \%$ & $16,250 \%$ & $11,963 \%$ & $5,828 \%$ & $2,438 \%$ \\
Pyrenophora & $0,024 \%$ & $0,003 \%$ & $0,180 \%$ & $0,485 \%$ & $0,436 \%$ \\
\hline $\begin{array}{l}\text { Puccinia } \\
\text { Filobasidium }\end{array}$ & $0,163 \%$ & $0,256 \%$ & $0,506 \%$ & $0,118 \%$ & $0,184 \%$ \\
\hline Stemphylium & $23,106 \%$ & $23,099 \%$ & $25,668 \%$ & $0,308 \%$ & $0,093 \%$ \\
\hline
\end{tabular}

\section{Olea}

\begin{tabular}{l|rcccc|}
\hline \multirow{2}{*}{ Taxon } & \multicolumn{5}{c}{ Relative abundance } \\
\cline { 2 - 6 } & S1 & S3 & S4 & S5 & S6 \\
\hline Epicoccum & $1,371 \%$ & $1,614 \%$ & $0,003 \%$ & $0,007 \%$ & $0,052 \%$ \\
Malassezia & $1,240 \%$ & $0,886 \%$ & $0,035 \%$ & $0,055 \%$ & $0,031 \%$ \\
Ustilaginales & $1,939 \%$ & $1,406 \%$ & & & \\
Didymella & $0,634 \%$ & $0,571 \%$ & $0,003 \%$ & & $0,007 \%$ \\
Mycosphaerella & $3,251 \%$ & $4,228 \%$ & $11,195 \%$ & $14,619 \%$ & $17,275 \%$ \\
Mycosphaerellaceae & $0,132 \%$ & $0,149 \%$ & $0,042 \%$ & $0,024 \%$ & $0,031 \%$ \\
Dothideomycetes & $0,152 \%$ & $0,218 \%$ & $0,533 \%$ & $0,800 \%$ & $0,880 \%$ \\
Celerioriella & & & $0,883 \%$ & $0,322 \%$ & $0,741 \%$ \\
Unidentified Fungi & $0,516 \%$ & $0,485 \%$ & $2,237 \%$ & $1,430 \%$ & $1,326 \%$ \\
Pseudocercospora & $0,017 \%$ & $0,052 \%$ & $2,888 \%$ & $1,534 \%$ & $2,902 \%$ \\
Cladosporium & $17,524 \%$ & $17,801 \%$ & $9,127 \%$ & $7,521 \%$ & $3,916 \%$ \\
Phoma & & & $0,602 \%$ & $0,592 \%$ & $0,485 \%$ \\
\hline
\end{tabular}

\section{Vitis}

\begin{tabular}{l|rcccc}
\hline \multirow{2}{*}{ Taxon } & \multicolumn{5}{c}{ Relative abundance } \\
\cline { 2 - 6 } & S1 & S3 & S4 & S5 & S6 \\
\hline Unidentified Fungi & $0,132 \%$ & $0,073 \%$ & $0,828 \%$ & $0,346 \%$ & $0,336 \%$ \\
Epicoccum & $0,980 \%$ & $0,696 \%$ & $0,003 \%$ & & $0,024 \%$ \\
Mycosphaerellaceae & $0,246 \%$ & $0,242 \%$ & $0,007 \%$ & & \\
Vishniacozyma & $0,218 \%$ & $0,869 \%$ & $4,477 \%$ & $2,417 \%$ & $2,348 \%$ \\
Cladosporium & $42,310 \%$ & $41,524 \%$ & $7,213 \%$ & $13,355 \%$ & $11,652 \%$ \\
Dothideomycetes & $0,080 \%$ & $0,073 \%$ & $0,069 \%$ & $0,564 \%$ & $0,433 \%$ \\
Mycosphaerella & $16,288 \%$ & $15,990 \%$ & $39,924 \%$ & $47,843 \%$ & $51,489 \%$ \\
Pyrenophora & $0,336 \%$ & $0,329 \%$ & $0,589 \%$ & $0,405 \%$ & $0,291 \%$ \\
Cystofilobasidium & $0,135 \%$ & $0,080 \%$ & $0,017 \%$ & $0,024 \%$ & \\
Cryptococcus & $0,010 \%$ & $0,107 \%$ & $0,554 \%$ & $0,651 \%$ & $0,488 \%$ \\
Puccinia & $1,229 \%$ & $0,803 \%$ & $1,468 \%$ & $0,038 \%$ & $0,003 \%$ \\
Ramularia & $0,014 \%$ & $0,010 \%$ & $0,190 \%$ & $0,222 \%$ & $0,197 \%$ \\
\hline
\end{tabular}

Fig. 6. Heatmaps showing taxa detected with a significantly different relative abundance (RA) using different primer sets (S1, S3, S4, S5, and S6) in each investigated host (Citrus, Olea, Triticum, and Vitis). Only taxa with an RA $\geq 0.05 \%$ were considered. The heatmap gradient is spread in three different colors, from dark green to dark red, from lowest to highest RA, respectively, passing through intermediate yellow degrees. White boxes indicate undetected taxa. 
level, the global data indicated a higher incidence of Ascomycota and a lower RA of Basidiomycota using ITS2 primers compared with primer targeting the ITS1 region. An exception was the primer set S4 targeting the ITS2 region that produced results more closely related to ITS1 primers. This described trend was particularly evident in Triticum. Regarding early divergent phyla (Zygomycota, Glomeromycota, and Chytridiomycota) a very low RA was detected with all primer sets. Only members of the phylum Zygomycota showed a generally higher incidence with ITS 1 primers in all hosts, particularly in Olea. The consistence of results obtained with different primer sets may represent an indirect confirmation of the actual low abundance of these taxa on plant leaves. However, reported issues relating to the amplification of ITS regions from these taxa, may have contributed to their low RA (Ishii et al. 2015). In fact, other regions such as the small or large ribosomal subunits, which are more conserved across the fungal kingdom, have been recently suggested as more appropriate targets for these taxa (Asemaninejad et al. 2016; Ishii and Fukui 2001; Toju et al. 2012).

Several fungal taxa were preferentially or exclusively detected by some primer sets and this association between primers and taxa was generally recurrent on more investigated hosts. For instance, taxa like Cladosporium, Didymella, Ustilaginales, and Epicoccum were preferentially detected by ITS1 primers, while others like Mycosphaerella, Togniniella, Celerioriella, and Pseudocercospora were mostly recovered by ITS2 primers. Interestingly, this sort of complementarity revealed between taxa suggest that a combination of primers targeting different regions may be useful to improve the fungal coverage. On Triticum, the genera Puccinia and Ustilago remained completely undetected with primer sets S5 and S6 while they represented a substantial part of the detected fungi with other primers. This result was likely a consequence of the forward primers (58A1F and ITS3-F_KYO2) since they are known for a low affinity with fungi of the class Pucciniomycetes (Tedersoo et al. 2015). In agreement with our results, a number of similar metagenomic studies, focused on mock communities, proved to have great difficulty in recovering the expected genetic diversity and/or in reflecting proportionally the amount of DNA of taxa included in the mix (Bellemain et al. 2010; Bokulich and Mills 2013; Comeau et al. 2017; De Filippis et al. 2017). Although the detection efficiency of different taxa is undoubtedly mainly related to the homology and affinity of primers with target regions, other factors cannot be excluded. For instance, the DNA extraction efficiency may be greatly influenced by the fungal species and organs, which is particularly difficult for resting spores (Lees et al. 2012). Furthermore, some taxa may be under- or overrepresented because of the nature of the rDNA that can vary in terms of copy numbers, greatly complicating the accurate estimation of the fungal abundance (Ganley and Kobayashi 2007; Taylor et al. 2016).

In conclusion, the present study provides new valuable experimental data on the impact of the choice of primers on the results of fungal metabarcoding analyses. An accurate knowledge of the specific features of different primers is important to enable the selection of the most suitable according to the host, the environment and the objective of the analysis. For instance, a higher level of specificity typical of ITS 1 primers may be particularly important for samples characterized by a very low percentage of target DNA as can be the case of plant fungal endophytes. On the other hand, the choice of a less specific primer, such as primer set S6 targeting the ITS2 region, may be useful in environmental samples where the coamplification of nontarget plant DNA is a secondary issue. Finally, primer set $\mathrm{S} 4$ may be an acceptable compromise for several applications since it combines features of primers binding the ITS2 region with a high level of specificity typical of ITS1 primers. Since the goal of defining a unique barcode for all fungal species and applications is not realistic, future investigation should also consider the opportunity of combining different target regions in order to cover most fungal lineages and have a picture of the fungal diversity as close as possible to the reality (Stielow et al. 2015). A major challenge in this direction will be the development of comprehensive and highly reliable databases (Abdelfattah et al. 2017).

\section{ACKNOWLEDGMENTS}

We thank A. Davies for the revision of the English text.

\section{LITERATURE CITED}

Abarenkov, K., Nilsson, R. H., Larsson, K. H., Alexander, I. J., Eberhardt, U., Erland, S., Høiland, K., Kjøller, R., Larsson, E., and Pennanen, T. 2010. The UNITE database for molecular identification of fungi-Recent updates and future perspectives. New Phytol. 186:281-285.

Abdelfattah, A., Malacrinò, A., Wisniewski, M., Cacciola, S. O., and Schena, L. 2017. Metabarcoding: A powerful tool to investigate microbial communities and shape future plant protection strategies. Biol. Control 120.

Altschul, S. F., Madden, T. L., Schäffer, A. A., Zhang, J., Zhang, Z., Miller, W., and Lipman, D. J. 1997. Gapped BLAST and PSI-BLAST: A new generation of protein database search programs. Nucleic Acids Res. 25:3389-3402.

Andrews, S. 2010. FastQC: A quality control tool for high throughput sequence data. Babraham Bioinformatics. https://www.bioinformatics.babraham.ac.uk/ projects/fastqc/

Asemaninejad, A., Weerasuriya, N., Gloor, G. B., Lindo, Z., and Thorn, G. 2016. New primers for discovering fungal diversity using nuclear large ribosomal DNA. PLoS One 11:e0159043.

Bálint, M., Schmidt, P. A., Sharma, R., Thines, M., and Schmitt, I. 2014. An Illumina metabarcoding pipeline for fungi. Ecol. Evol. 4:2642-2653.

Bellemain, E., Carlsen, T., Brochmann, C., Taberlet, P., and Kauserud, H. 2010. ITS as an environmental DNA barcode for fungi: An in silico approach reveals potential PCR biases. BMC Microbiol. 10:189.

Blaalid, R., Kumar, S., Nilsson, R. H., Abarenkov, K., Kirk, P. M., and Kauserud, H. 2013. ITS1 versus ITS2 as DNA metabarcodes for fungi. Mol. Ecol. Resour. 13:218-224.

Blackwell, M. 2011. The fungi: 1, 2, 3 ... 5.1 million species? Am. J. Bot. 98: 426-438.

Bokulich, N. A., and Mills, D. A. 2013. Improved selection of internal transcribed spacer-specific primers enables quantitative, ultra-high-throughput profiling of fungal communities. Appl. Environ. Microbiol. 79:2519-2526.

Bruns, T. D., White, T. J., and Taylor, J. W. 1991. Fungal molecular systematics. Annu. Rev. Ecol. Syst. 22:525-564.

Caporaso, J. G., Kuczynski, J., Stombaugh, J., Bittinger, K., Bushman, F. D., Costello, E. K., Fierer, N., Pena, A. G., Goodrich, J. K., and Gordon, J. I. 2010. QIIME allows analysis of high-throughput community sequencing data. Nat. Methods 7:335-336.

Cheng, T., Xu, C., Lei, L., Li, C., Zhang, Y., and Zhou, S. 2016. Barcoding the kingdom Plantae: New PCR primers for ITS regions of plants with improved universality and specificity. Mol. Ecol. Resour. 16:138-149.

Comeau, A. M., Douglas, G. M., and Langille, M. G. I. 2017. Microbiome helper: A custom and streamlined workflow for microbiome research. mSystems 2:e00127-e16.

De Filippis, F., Laiola, M., Blaiotta, G., and Ercolini, D. 2017. Different amplicon targets for sequencing-based studies of fungal diversity. Appl. Environ. Microbiol. 83:e00905-e00917.

Ficetola, G. F., Coissac, E., Zundel, S., Riaz, T., Shehzad, W., Bessière, J., Taberlet, P., and Pompanon, F. 2010. An in silico approach for the evaluation of DNA barcodes. BMC Genomics 11:434.

Ganley, A. R. D., and Kobayashi, T. 2007. Highly efficient concerted evolution in the ribosomal DNA repeats: Total rDNA repeat variation revealed by whole-genome shotgun sequence data. Genome Res. 17:184-191.

Gardes, M., and Bruns, T. D. 1993. ITS primers with enhanced specificity for basidiomycetes-Application to the identification of mycorrhizae and rusts. Mol. Ecol. 2:113-118.

Halwachs, B., Madhusudhan, N., Krause, R., Nilsson, R. H., Moissl-Eichinger, C., Högenauer, C., Thallinger, G. G., and Gorkiewicz, G. 2017. Critical issues in mycobiota analysis. Front. Microbiol. 8:180.

Hawksworth, D. L. 1991. The fungal dimension of biodiversity: Magnitude, significance, and conservation. Mycol. Res. 95:641-655. 
He, Y., Caporaso, J. G., Jiang, X.-T., Sheng, H.-F., Huse, S. M., Rideout, J. R., Edgar, Robert C., Henrik Nilsson, R., Tedersoo, L., Lindahl, B. D., Kjøller, R., Carlsen, T., Quince, C., Abarenkov, K., Pennanen, T., Stenlid, J., Bruns, T., Larsson, K. H., Kõljalg, U., and Kauserud, H. 2011. Towards standardization of the description and publication of next-generation sequencing datasets of fungal communities. New Phytol. 191:314-318.

Ihrmark, K., Bödeker, I. T. M., Cruz-Martinez, K., Friberg, H., Kubartova, A., Schenck, J., Strid, Y., Stenlid, J., Brandström-Durling, M., Clemmensen, K. E., and Lindahl, B. D. 2012. New primers to amplify the fungal ITS2 region-evaluation by 454-sequencing of artificial and natural communities. FEMS Microbiol. Ecol. 82:666-677.

Ishii, K., and Fukui, M. 2001. Optimization of annealing temperature to reduce bias caused by a primer mismatch in multitemplate PCR. Appl. Environ. Microbiol. 67:3753-3755.

Ishii, N., Ishida, S., and Kagami, M. 2015. PCR primers for assessing community structure of aquatic fungi including Chytridiomycota and Cryptomycota. Fungal Ecol. 13:33-43.

Kõljalg, U., Nilsson, R. H., Abarenkov, K., Tedersoo, L., Taylor, A. F. S., Bahram, M., Bates, S. T., Bruns, T. D., Bengtsson-Palme, J., Callaghan, T. M., Douglas, B., Drenkhan, T., Eberhardt, U., Dueñas, M., Grebenc, T., Griffith, G. W., Hartmann, M., Kirk, P. M., Kohout, P., Larsson, E., Lindahl, B. D., Lücking, R., Martín, M. P., Matheny, P. B., Nguyen, N. H., Niskanen, T., Oja, J., Peay, K. G., Peintner, U., Peterson, M., Põldmaa, K., Saag, L., Saar, I., Schüßler, A., Scott, J. A., Senés, C., Smith, M. E., Suija, A., Taylor, D. L., Telleria, M. T., Weiss, M., and Larsson, K. H. 2013. Towards a unified paradigm for sequence-based identification of fungi. Mol. Ecol. 22: 5271-5277.

Kumar, S., Stecher, G., and Tamura, K. 2016. MEGA7: Molecular Evolutionary Genetics Analysis version 7.0 for bigger datasets. Mol. Biol. Evol. 33: 1870-1874.

Lees, A. K., Sullivan, L., Lynott, J. S., and Cullen, D. W. 2012. Development of a quantitative real-time PCR assay for Phytophthora infestans and its applicability to leaf, tuber and soil samples. Plant Pathol. 61:867-876.

Lindahl, B. D., Nilsson, R. H., Tedersoo, L., Abarenkov, K., Carlsen, T., Kjøller, R., Kõljalg, U., Pennanen, T., Rosendahl, S., Stenlid, J., and Kauserud, H. 2013. Fungal community analysis by high-throughput sequencing of amplified markers-A user's guide. New Phytol. 199:288-299.

Martin, K. J., and Rygiewicz, P. T. 2005. Fungal-specific PCR primers developed for analysis of the ITS region of environmental DNA extracts. BMC Microbiol. 5:28.

Mueller, R. C., Gallegos-Graves, L. V., and Kuske, C. R. 2016. A new fungal large subunit ribosomal RNA primer for high-throughput sequencing surveys. FEMS Microbiol. Ecol. 92:1-11.

Nilsson, R. H., Ryberg, M., Abarenkov, K., Sjökvist, E., and Kristiansson, E. 2009. The ITS region as a target for characterization of fungal communities using emerging sequencing technologies. FEMS Microbiol. Letts. 296: 97-101.

Op De Beeck, M., Lievens, B., Busschaert, P., Declerck, S., Vangronsveld, J., and Colpaert, J. V. 2014. Comparison and validation of some ITS primer pairs useful for fungal metabarcoding studies. PLoS One 9:e97629.

Orgiazzi, A., Lumini, E., Nilsson, R. H., Girlanda, M., Vizzini, A., Bonfante, P., and Bianciotto, V. 2012. Unravelling soil fungal communities from different Mediterranean land-use backgrounds. PLoS One 7:e34847.

Porter, T. M., and Golding, B. 2011. Are similarity- or phylogeny-based methods more appropriate for classifying internal transcribed spacer (ITS) metagenomic amplicons? New Phytol. 192:775-782.

Rognes, T., Flouri, T., Nichols, B., Quince, C., and Mahé, F. 2016. VSEARCH: A versatile open source tool for metagenomics. PeerJ 4:e2584.

Schoch, C. L., Robbertse, B., Robert, V., Vu, D., Cardinali, G., Irinyi, L., Meyer, W., Nilsson, R. H., Hughes, K., Miller, A. N., Kirk, P. M., Abarenkov, K., Aime, M. C., Ariyawansa, H. A., Bidartondo, M., Boekhout, T., Buyck, B., Cai, Q., Chen, J., Crespo, A., Crous, P. W., Damm, U., De Beer, Z. W., Dentinger, B. T. M., Divakar, P. K., Dueñas, M., Feau, N., Fliegerova, K., García, M. A., Ge, Z. W., Griffith, G. W., Groenewald, J. Z., Groenewald, M., Grube, M., Gryzenhout, M., Gueidan, C., Guo, L., Hambleton, S., Hamelin, R., Hansen, K., Hofstetter, V., Hong, S. B., Houbraken, J., Hyde, K. D., Inderbitzin, P., Johnston, P. R., Karunarathna, S. C., Kõljalg, U., Kovács, G. M., Kraichak, E., Krizsan, K., Kurtzman, C. P., Larsson, K. H., Leavitt, S., Letcher, P. M., Liimatainen, K., Liu, J. K., Lodge, D. J., Luangsa-Ard, J. J., Lumbsch, H. T., Maharachchikumbura, S. S.N., Manamgoda, D., Martín, M. P., Minnis, A. M., Moncalvo, J. M., Mulè, G., Nakasone, K. K., Niskanen, T., Olariaga, I., Papp, T., Petkovits, T., Pino-Bodas, R., Powell, M. J., Raja, H. A., Redecker, D., Sarmiento-Ramirez, J. M., Seifert, K. A., Shrestha, B., Stenroos, S., Stielow, B., Suh, S. O., Tanaka, K., Tedersoo, L., Telleria, M. T.,
Udayanga, D., Untereiner, W. A., Uribeondo, J. D., Subbarao, K. V., Vágvölgyi, C., Visagie, C., Voigt, K., Walker, D. M., Weir, B. S., Weiß, M., Wijayawardene, N. N., Wingfield, M. J., Xu, J. P., Yang, Z. L., Zhang, N., Zhuang, W. Y., and Federhen, S. 2014. Finding needles in haystacks: Linking scientific names, reference specimens and molecular data for Fungi. Database, Volume 2014:bau061.

Schoch, C. L., Seifert, K. A., Huhndorf, S., Robert, V., Spouge, J. L., Levesque, C. A., Chen, W., Bolchacova, E., Voigt, K., Crous, P. W., Miller, A. N., Wingfield, M. J., Aime, M. C., An, K. D., Bai, F. Y., Barreto, R. W., Begerow, D., Bergeron, M. J., Blackwell, M., Boekhout, T., Bogale, M., Boonyuen, N., Burgaz, A. R., Buyck, B., Cai, L., Cai, Q., Cardinali, G., Chaverri, P., Coppins, B. J., Crespo, A., Cubas, P., Cummings, C., Damm, U., de Beer, Z. W., de Hoog, G. S., Del-Prado, R., Dentinger, B., Dieguez-Uribeondo, J., Divakar, P. K., Douglas, B., Duenas, M., Duong, T. A., Eberhardt, U.,

Edwards, J. E., Elshahed, M. S., Fliegerova, K., Furtado, M., Garcia, M. A., Ge, Z. W., Griffith, G. W., Griffiths, K., Groenewald, J. Z., Groenewald, M., Grube, M., Gryzenhout, M., Guo, L. D., Hagen, F., Hambleton, S., Hamelin, R. C., Hansen, K., Harrold, P., Heller, G., Herrera, C., Hirayama, K., Hirooka, Y., Ho, H. M., Hoffmann, K., Hofstetter, V., Hognabba, F., Hollingsworth, P. M., Hong, S. B., Hosaka, K., Houbraken, J., Hughes, K., Huhtinen, S., Hyde, K. D., James, T., Johnson, E. M., Johnson, J. E., Johnston, P. R., Jones, E. B. G., Kelly, L. J., Kirk, P. M., Knapp, D. G., Koljalg, U., Kovacs, G. M., Kurtzman, C. P., Landvik, S., Leavitt, S. D., Liggenstoffer, A. S., Liimatainen, K., Lombard, L., Luangsa-ard, J. J., Lumbsch, H. T., Maganti, H., Maharachchikumbura, S. S. N., Martin, M. P., May, T. W., McTaggart, A. R., Methven, A. S., Meyer, W., Moncalvo, J. M., Mongkolsamrit, S., Nagy, L. G., Nilsson, R. H., Niskanen, T., Nyilasi, I., Okada, G., Okane, I., Olariaga, I., Otte, J., Papp, T., Park, D., Petkovits, T., Pino-Bodas, R., Quaedvlieg, W., Raja, H. A., Redecker, D., Rintoul, T. L., Ruibal, C., Sarmiento-Ramirez, J. M., Schmitt, I., Schussler, A., Shearer, C., Sotome, K., Stefani, F. O. P., Stenroos, S., Stielow, B., Stockinger, H., Suetrong, S., Suh, S. O., Sung, G. H., Suzuki, M., Tanaka, K., Tedersoo, L., Telleria, M. T., Tretter, E., Untereiner, W. A., Urbina, H., Vagvolgyi, C., Vialle, A., Vu, T. D., Walther, G., Wang, Q. M., Wang, Y., Weir, B. S., Weiss, M., White, M. M., Xu, J., Yahr, R., Yang, Z. L., Yurkov, A., Zamora, J. C., Zhang, N., Zhuang, W. Y., and Schindel, D. 2012. Nuclear ribosomal internal transcribed spacer (ITS) region as a universal DNA barcode marker for Fungi. Proc. Natl. Acad. Sci. 109:6241-6246.

Stielow, J. B., Lévesque, C. A., Seifert, K. A., Meyer, W., Irinyi, L., Smits, D., Renfurm, R., Verkley, G. J. M., Groenewald, M., Chaduli, D., Lomascolo, A., Welti, S., Lesage-Meessen, L., Favel, A., Al-Hatmi, A. M. S., Damm, U., Yilmaz, N., Houbraken, J., Lombard, L., Quaedvlieg, W., Binder, M., Vaas, L. A. I., Vu, D., Yurkov, A., Begerow, D., Roehl, O., Guerreiro, M., Fonseca, A., Samerpitak, K., van Diepeningen, A. D., Dolatabadi, S., Moreno, L. F., Casaregola, S., Mallet, S., Jacques, N., Roscini, L., Egidi, E., Bizet, C.,

Garcia-Hermoso, D., Martín, M. P., Deng, S., Groenewald, J. Z., Boekhout, T., de Beer, Z. W., Barnes, I., Duong, T. A., Wingfield, M. J., de Hoog, G. S., Crous, P. W., Lewis, C. T., Hambleton, S., Moussa, T. A. A., Al-Zahrani, H. S., Almaghrabi, O. A., Louis-Seize, G., Assabgui, R., McCormick, W., Omer, G., Dukik, K., Cardinali, G., Eberhardt, U., de Vries, M., and Robert, V. 2015. One fungus, which genes? Development and assessment of universal primers for potential secondary fungal DNA barcodes.Persoonia-Mol. Phylogeny Evol. Fungi. 35:242-263.

Taylor, D. L., Hollingsworth, T. N., McFarland, J. W., Lennon, N. J., Nusbaum, C., and Ruess, R. W. 2014. A first comprehensive census of fungi in soil reveals both hyperdiversity and fine-scale niche partitioning. Ecol. Monogr. 84:3-20.

Taylor, D. L., Walters, W. A., Lennon, N. J., Bochicchio, J., Krohn, A., Caporaso, J. G., and Pennanen, T. 2016. Accurate estimation of fungal diversity and abundance through improved lineage-specific primers optimized for Illumina amplicon sequencing. Appl. Environ. Microbiol. 82: 7217-7226.

Tedersoo, L., Anslan, S., Bahram, M., Põlme, S., Riit, T., Liiv, I., Kõljalg, U., Kisand, V., Nilsson, H., Hildebrand, F., Bork, P., and Abarenkov, K. 2015. Shotgun metagenomes and multiple primer pair-barcode combinations of amplicons reveal biases in metabarcoding analyses of fungi. MycoKeys 10: $1-43$.

Tedersoo, L., Bahram, M., Polme, S., Koljalg, U., Yorou, N. S., Wijesundera, R., Ruiz, L. V., Vasco-Palacios, A. M., Thu, P. Q., Suija, A., Smith, M. E., Sharp, C., Saluveer, E., Saitta, A., Rosas, M., Riit, T., Ratkowsky, D., Pritsch, K., Poldmaa, K., Piepenbring, M., Phosri, C., Peterson, M., Parts, K., Partel, K., Otsing, E., Nouhra, E., Njouonkou, A. L., Nilsson, R. H., Morgado, L. N., Mayor, J., May, T. W., Majuakim, L., Lodge, D. J., Lee, S. S., Larsson, K. H., Kohout, P., Hosaka, K., Hiiesalu, I., Henkel, T. W., Harend, H., Guo, L. D., Greslebin, A., Grelet, G., Geml, J., Gates, G., Dunstan, W., Dunk, C., 
Drenkhan, R., Dearnaley, J., De Kesel, A., Dang, T., Chen, X., Buegger, F., Brearley, F. Q., Bonito, G., Anslan, S., Abell, S., and Abarenkov, K. 2014. Global diversity and geography of soil fungi. Science 346 : 1256688.

Toju, H., Tanabe, A. S., Yamamoto, S., and Sato, H. 2012. High-coverage ITS primers for the DNA-based identification of Ascomycetes and Basidiomycetes in environmental samples. PLoS One 7:e40863.

Turenne, C. Y., Sanche, S. E., Hoban, D. J., Karlowsky, J. A., and Kabani, A. M. 1999. Rapid identification of fungi by using the ITS2 genetic region and an automated fluorescent capillary electrophoresis system. J. Clin. Microbiol. 37: 1846-1851.

Ursell, L. K., Metcalf, J. L., Parfrey, L. W., and Knight, R. 2012. Defining the human microbiome. Nutr. Rev. 70:S38-S44.
Walters, W. A., Caporaso, J. G., Lauber, C. L., Berg-Lyons, D., Fierer, N., and Knight, R. 2011. PrimerProspector: De novo design and taxonomic analysis of barcoded polymerase chain reaction primers. Bioinformatics 27: 1159-1161.

Waud, M., Busschaert, P., Ruyters, S., Jacquemyn, H., and Lievens, B. 2014. Impact of primer choice on characterization of orchid mycorrhizal communities using 454 pyrosequencing. Mol. Ecol. Resour. 14:679-699.

White, T. J., Bruns, S., Lee, S., and Taylor, J. 1990. Amplification and Direct Sequencing of Fungal Ribosomal RNA Genes for Phylogenetics. Pages 315-322 in: PCR Protocols: A Guide to Methods and Applications. Academic Press, New York. $\mathrm{Xu}$, J. 2016. Fungal DNA barcoding. Genome 59:913-932.

Zhang, J., Kobert, K., Flouri, T., and Stamatakis, A. 2014. PEAR: A fast and accurate Illumina Paired-End reAd mergeR. Bioinformatics 30:614-620. 